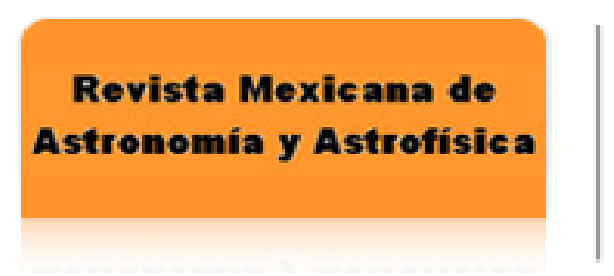

Revista Mexicana de Astronomía y Astrofísica ISSN: 0185-1101

rmaa@astroscu.unam.mx

Instituto de Astronomía

México

Galván-Madrid, R.; Zhang, Q.; Keto, E. R.; Rodríguez, L. F.; Ho, P. T. P.

ON THE FORMATION OF THE MOST MASSIVE STARS IN THE GALAXY

Revista Mexicana de Astronomía y Astrofísica, vol. 40, 2011, pp. 239-240

Instituto de Astronomía

Distrito Federal, México 
RevMexAA (Serie de Conferencias), 40, 239-240 (2011)

\title{
ON THE FORMATION OF THE MOST MASSIVE STARS IN THE GALAXY
}

\author{
R. Galván-Madrid, ${ }^{1,2,3}$ Q. Zhang, ${ }^{1}$ E. R. Keto, ${ }^{1}$ L. F. Rodríguez, ${ }^{2}$ and P. T. P. Ho ${ }^{1,3}$ \\ RESUMEN
}

Presentamos resultados obtenidos con el Submillimeter Array (SMA) y el Very Large Array (VLA) en regiones de formación de estrellas masivas en sus primeras etapas de ionización. Las observaciones de gas ionizado y molecular con resolución angular de hasta $0.3^{\prime \prime}$ revelan rotación, colapso, y flujos eyectados en escalas de parsecs a $<0.05$ pc. Los lugares con formación de estrellas tienen varias protoestrellas masivas en diferentes estados evolutivos. Los resultados indican que: (i) Las estrellas con $M_{\star}>15 M_{\odot}$ se forman por procesos de acreción similares a los de estrellas de baja masa, pero con ionización considerable. (ii) Los núcleos moleculares a partir de los cuales se forman estas estrellas continúan acretando gas de su ambiente. (iii) La acreción a la estrella puede continuar después del inicio de la ionización.

\section{ABSTRACT}

We present results of Submillimeter Array (SMA) and Very Large Array (VLA) studies of massive-star formation regions in the early stages of ionization. Molecular-line and ionized-gas observations at resolutions down to $0.3^{\prime \prime}$ reveal the presence of rotation, infall, and/or outflow from parsec scales to $<0.05 \mathrm{pc}$. The centers of star formation harbor small groups of massive (proto)stars at different evolutionary stages. These observations favor a picture in which: (i) Stars with $M_{\star}>15 M_{\odot}$ form by accretion processes similar to those of lower-mass stars, but with significant ionization. (ii) The molecular cores from which these stars form continue accreting from their environment. (iii) Accretion to the star can continue past the onset of ionization.

Key Words: H II regions - ISM: individual objects (W33A, G12.91-0.26, G20.08-0.14) — stars: formation

\section{INTRODUCTION}

To study the accretion dynamics over a range of scales in massive star formation regions (MSFRs), from the cluster scales $(>1 \mathrm{pc})$ down to $<0.05 \mathrm{pc}$, we set up a program of molecular line, recombination line, and continuum observations using the Submillimeter Array (SMA) and the Very Large Array (VLA) with several different angular resolutions. We chose exclusively sources with bolometric luminosities $L>10^{5} L_{\odot}$ to ensure that they are forming (or can form) O-type stars $\left(M_{\star}>15\right.$ to $\left.20 M_{\odot}\right)$. Here we summarize results of two sources representative of the low- and high-ionization ends of our sample. We refer the reader to Galván-Madrid et al. (2009, 2010) for further details.

\section{W33A}

W33A (G12.91-0.26, $\left.L \approx 10^{5} L_{\odot}\right)$ is a region with very high luminosity but very faint radiocontinuum emission (Stier et al. 1984). We present

\footnotetext{
${ }^{1}$ Harvard-Smithsonian Center for Astrophysics, USA (rgalvan@cfa.harvard.edu).

${ }^{2}$ Centro de Radioastronomía y Astrofísica, Universidad Nacional Autónoma de México, Mexico.

${ }^{3}$ Academia Sinica, Institute of Astronomy and Astrophysics, Taiwan.
}

the first resolved observations in both mm continuum and molecular-line emission of this MSFR. The two previously known mm cores (MM1 and MM2, see Figure 1) are fragmented into multiple peaks, and are at different evolutionary stages, as indicated by their molecular spectra, masses, temperatures, and continuum spectral indices. The warm-gas dynamics around the brightest core (MM1-Main at the center of MM1) indicates the presence of a circumstellar disk plus envelope rotating around a stellar mass of $M_{\star} \approx 10 M_{\odot}$. MM1-Main also drives a powerful, high-velocity molecular outflow perpendicular to the disk. MM2, the coldest and most massive core, is not detected in hot-core lines but appears to drive a more modest outflow. Both MM1 and MM2 are located at the intersection of parsec-scale filamentary structures with a line-of-sight velocity offset of $\approx 2.6 \mathrm{~km} \mathrm{~s}^{-1}$. The position-position-velocity structure of these filaments (see Figure 1) and a comparison with recent numerical simulations by VázquezSemadeni et al. (2009) suggest that star formation in W33A was triggered by the convergence of filaments of cold molecular gas.

\section{G20.08N}

G20.08-0.14 North (G20.08N, $\left.L \approx 6.6 \times 10^{5} L_{\odot}\right)$ was identified in the $\mathrm{H}$ II region VLA survey of Wood 

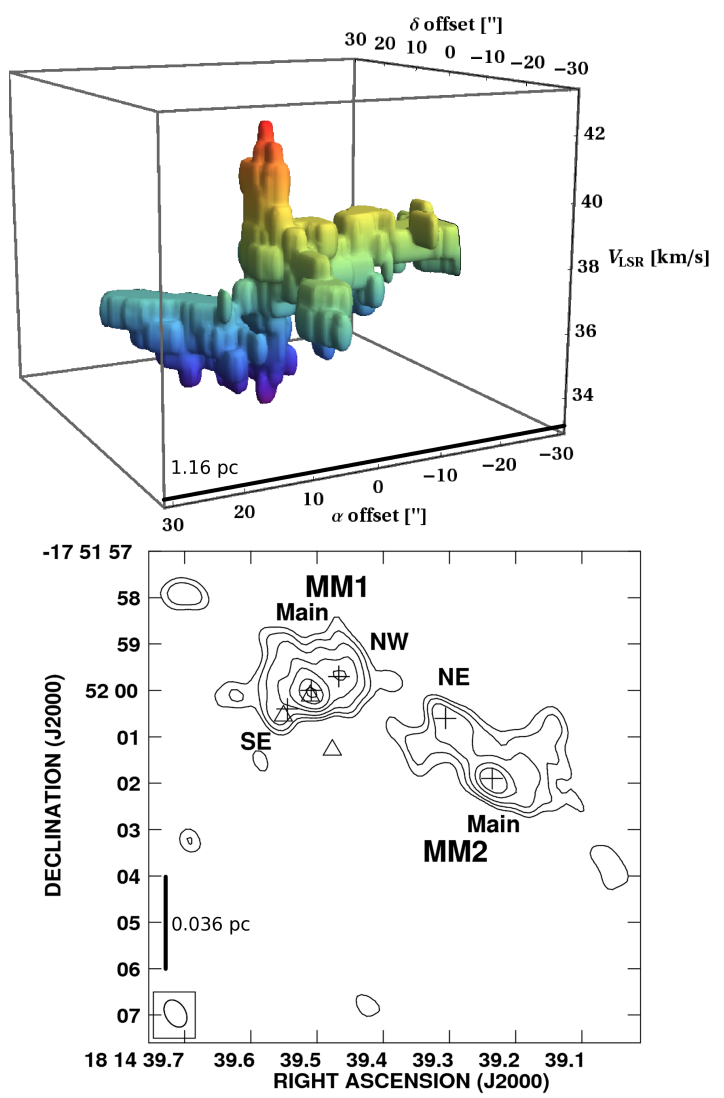

Fig. 1. Top: position-position-velocity rendering of the parsec-scale filaments observed in the $\mathrm{NH}_{3}(2,2)$ data toward W33A. The two filamentary structures at different velocities do not merely superpose at the position center, but merge in position-position-velocity space, suggesting interaction of the filaments. Bottom: continuum at $231 \mathrm{GHz}(1.3 \mathrm{~mm})$ from the SMA Compact+VEX data at the intersection of the filaments shown in the top panel. The dusty star-forming cores are labeled.

\& Churchwell (1989). We find a large-scale (radius $\sim 0.5 \mathrm{pc}$ ) accretion flow around and into a star cluster with several O-type stars, identified by one UC and two HC H II regions. This flow is rotating (Figure 2 )and infalling towards its center. The two HC H II regions have infall toward them as inferred from subarcsecond resolution $\mathrm{NH}_{3}$ observations. The brightest $\mathrm{HC} \mathrm{H}$ II region (A) is detected in mm emission lines that trace warm molecular gas. This gas rotates in concordance with the large-scale flow (Figure 2). The presence of rotation and infall at both scales $(\sim 0.5 \mathrm{pc}$ and $\sim 0.05 \mathrm{pc})$, as well as the short dynamical time-scale of the small-scale flow, suggest that the small cores are still accreting from their environment. This result supports models of star for-

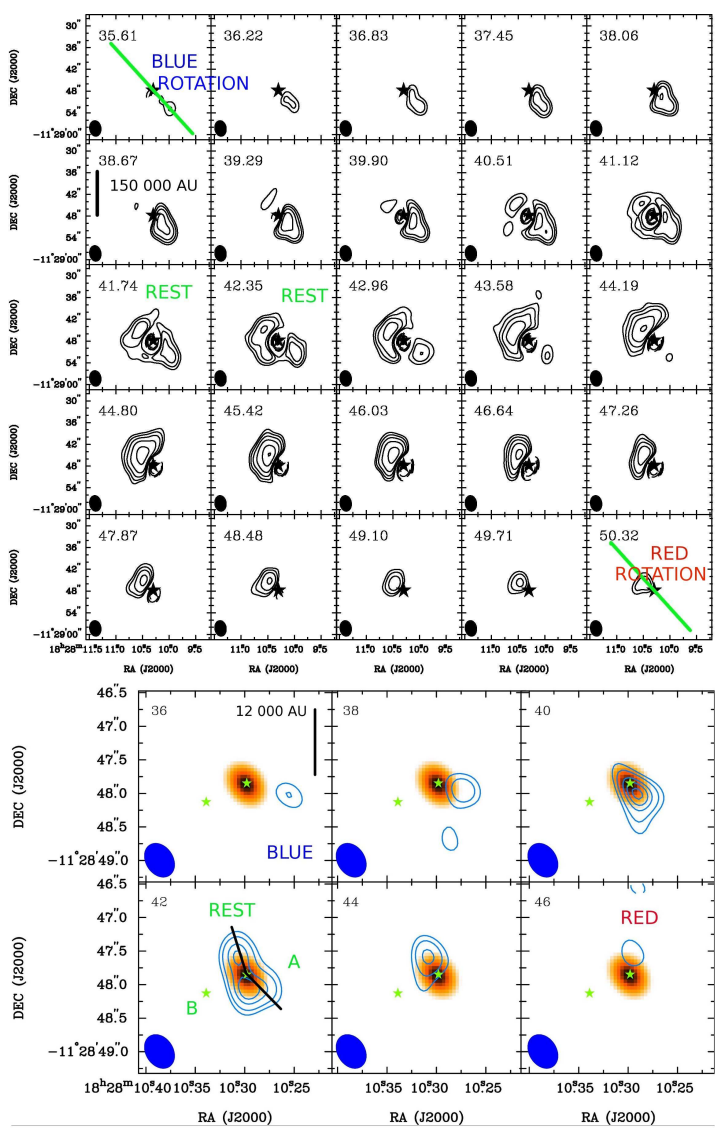

Fig. 2. Top: $\mathrm{NH}_{3}(3,3)$ observations of the pc-scale emission. The star covers the three UC and HC H II regions mentioned in the text. Estimates of the gas mass ( $M_{\text {gas }} \sim 2000 M_{\odot}$, from molecular lines and dust) and stellar mass $\left(M_{\star} \approx 34 M_{\odot}\right.$, from the continuum SED) indicate that the velocity gradient is caused by rotation ( $\mathrm{a} \approx 2 \mathrm{~km} \mathrm{~s}^{-1}$ infall component is also detected from absorption). Bottom: warm gas emission traced by $\mathrm{CH}_{3} \mathrm{CN}$ 12-11 (contours) around the HC H II region A (color). The rotation motions in the inner $0.1 \mathrm{pc}$ are similar to those in the pc-scale flow.

mation initiated by global collapse of the parental cloud (Field et al. 2008; Vázquez-Semadeni et al. 2009; Wang et al. 2010; Peters et al. 2010).

\section{REFERENCES}

Field, G., et al. 2008, MNRAS, 385, 181

Galván-Madrid, R., et al. 2009, ApJ, 706, 1036

Galván-Madrid, R. et al. 2010, ApJ, 725, 17

Peters, T., et al. 2010, ApJ, 711, 101

Stier, M. T., et al. 1984, ApJ, 283, 573

Vázquez-Semadeni, E., et al. 2009, ApJ, 707, 1023

Wang, P., et al. 2010, ApJ, 709, 27

Wood, D. O. S., \& Churchwell, E. 1989, ApJS, 69, 831 